\title{
La estética convulsiva: Beckett, Chaplin y Charcot
}

\author{
Ulrika Maude' \\ University of Bristol, Inglaterra \\ Traducción: Marcelo Lara
}

\begin{abstract}
El estilo distintivo de Samuel Beckett se desarrolló en un periodo de la historia europea que fue testigo de avances extraordinarios en el campo de la ciencia, la tecnología, la cultura y las artes. Beckett nació once años después del descubrimiento en 1895 de los rayos X, año en el que también Marconi inventó la telegrafía sin cable y en el que los hermanos Lumière proyectaron la primera película de la historia, quizás sin ser demasiado conscientes de que estaban inaugurando un nuevo arte. Una de las áreas de la ciencia que fue testigo de avances verdaderamente sin precedentes fue la medicina, $y$ en particular el campo de la neurología, en el que el descubrimiento de la sinapsis y de los circuitos neuronales hacia finales del siglo XIX transformó la comprensión de las funciones cerebrales. Particularmente importantes en el campo de la neurología fueron los descubrimientos de Santiago Ramón y Cajal (1852-1934), el histólogo español que inauguró lo que hoy es conocido como "teoría neuronal" y quien fue motivado por su búsqueda del "curso material del pensamiento y de la voluntad" (Porter 1999: 536). Igualmente importantes fueron, por un lado, el hallazgo de Paul Broca de la localización del lenguaje en la región anteroposterior de los lóbulos frontales -hoy conocido como el área de Broca-, y por el otro, sus estudios sobre la afasia de lenguaje, materializado en las formas en que los escritores modernistas se sintieron obligados a responder, presentando con frecuencia en sus trabajos una sintaxis rota similar al lenguaje patológico. Otra figura que influyó en el desarrollo de la ciencia del cerebro fue Jean-Martin Charcot. En 1882 Charcot fundó una clínica neurológica en el Hospital Salpêtrière en París; aquella fue la primera de esa especialidad en Europa, y entre los estudiantes de Charcot en la Salpêtrière se encontraban Sigmund Freud, Joseph Babinski, Pierre Janet, William James y George Gilles de la Tourette, sólo para nombrar sólo.
\end{abstract}

Durante este periodo de acelerado avance científico se observaron y nominaron varias enfermedades neurológicas. Estas incluían muchos desórdenes de movimiento involuntario o discinesia como el mal de Parkinson. La propia investigación que Charcot llevó a cabo entre 1868 y 1881 sobre lo que en su tiempo se conocía como "parálisis agitante" fue revolucionaria. Otra enfermedad minuciosamente investigada en el

1. Le agradecemos a Ulrica Maude y a Edinburgh University Press el permiso que nos han otorgado para traducir y publicar en Becketiana el artículo "Convulsive Aesthetics: Beckett, Chaplin and Charcot" (pp. 44-53), incluido en The Edinburgh Companion to Samuel Beckett and the Arts (2014) Ed. S. E. Gontarski. 
hospital Salpêtrière fue el síndrome de Tourette, observado por Charcot y Freud, pero investigado principalmente por Gilles de la Tourette (1857-1904), discípulo de Charcot, cuya descripción clínica se remonta a 1885. El mismo Charcot también se interesó por la histeria, a la que consideraba como un desorden neurológico. Aunque convencionalmente y "por ese mismo nombre se denomine una dolencia de la mujer", la histeria para el neurólogo no era meramente una aflicción femenina. Él argumentaba que la causa era hereditaria, en línea de pensamiento con las ideas decimonónicas sobre la degeneración o 'bad stock' (Hacking 1998: 22-3). Sin embargo, para Charcot los disparadores de la histeria en cada sexo eran diferentes: "las causas morales pueden incitar a la histeria femenina, pero en los hombres, a menos que el desorden hubiera aparecido cuando el paciente era muy joven, la causa principal parecía ser el trauma físico o shock" (ibíd.: 33). En el obituario que escribe Freud para Charcot, el padre del psicoanálisis elogia al neurólogo por haber tratado la histeria en los últimos diez años de su vida como un área de estudio serio, aunque su propia perspectiva sobre la enfermedad estaba basada en la noción de neurosis. ${ }^{2}$

El conocimiento de los descubrimientos neurológicos comenzó a popularizarse rápidamente. En Francia este fenómeno se produjo a través de las "performances" públicas de las histéricas sobre un escenario montado en el hospital de la Salpêtrière. Charcot "exhibía en sus famosas clases de los martes a una o más pacientes y desarrollaba los casos y sus síntomas ante la mirada llena de admiración de la multitud" (ibíd.: 35). El conocimiento sobre las enfermedades neurológicas también fue diseminado a través del diario Nouvelle Iconographie photographique de la Salpêtrière, en el que aparecían imágenes de histéricas y de epilépticos, así como también de otros pacientes con diversos desórdenes neurológicos. Esta práctica de exposición de casos se extendió desde 1888 hasta 1918, y tuvo a Gilles de la Tourette como uno de sus fundadores. Por otra parte, hacia finales del siglo XIX, "el nombre de la Salpêtrière invadió incluso las revistas y periódicos populares" (Micale 1995: 198), en este sentido, Georges Didi-Huberman ha argumentado que "[s]e identificó incluso, soterradamente, con una especie de manifestación artística. Un arte muy próximo al teatro y a la pintura" (Didi-Huberman 2007: 8). ${ }^{3}$ Por su parte, Beth Rae Gordon, quien ha investigado la influencia de la neurología en la cultura teatral francesa, argumenta que "El cabaret parisino y el café-concert entre 1865 y 1907 se caracterizaban por mostrar un lenguaje corporal convulsivo hecho de movimientos frenéticos, angulares y 'mecánicos' acompañados por tics y muecas'. La autora ha demostrado que en las postrimerías del siglo XIX las performances de cabaret en París estaban "muy influenciadas por el discurso médico sobre la histeria y la epilepsia y por las descripciones populares de los desórdenes nerviosos que aparecían en diarios y revistas" (Gordon 2004: 100).

Charcot clasificó los movimientos de las histéricas como convulsivos, pero sobre los epilépticos argumentó que exhibían movimientos clownescos: temblores, contracturas, asimetría facial, tics, muecas y agitación constante. La acatisia -incapacidad de mantenerse sentado- y la renguera eran otras patologías del movimiento asociadas con la histeria y la epilepsia. Estos síntomas subieron al escenario y más tarde a la pantalla del cine a través de los actores del cabaret y del music hall, quienes luego desarrollaron en el séptimo arte carreras artísticas muy exitosas. Muchos comediantes, como por ejemplo Dranem y Louise-Jacques Boucot en Francia, y Karl Valentin en Alemania -conocido como "el Charlie Chaplin alemán"- tomaron ese camino artístico. El director vanguardista Jean Epstein, de hecho, identificó el estilo de Charlie Chaplin (1889-1977) como un caso de "neurastenia fotogénica": "toda su actuación consiste en las acciones

2. Ver Sigmund Freud y Joseph Breuer, Estudios sobre la histeria, publicado por primera vez en 1895.

3. Utilizo la edición de Didi-Huberman, Georges (2007). La invención de la histeria. Charcot y la iconografía de la Salpêtrière. Trad. Tania Arias y Rafael Jackson. Madrid: Cátedra. 
reflejas de una persona nerviosa y cansada", escribió (Epstein 1998: 238). Chaplin por supuesto también inició su carrera actuando en los music halls de Londres en la troupe de mimos de Fred Karno, y "uno sólo tiene que comparar su modo de andar con el de los pacientes psiquiátricos filmados en la Salpêtrière entre 1910 y 1912" para observar la conexión entre Charcot y Charlot -el apodo francés Chaplin (Gordon 2004: 99). ${ }^{4} \mathrm{El}$ mismo Epstein había estudiado medicina antes de ser director de cine, y por lo tanto tenía la vista entrenada para leer la influencia de la clínica en la nueva forma de arte. Como hemos visto, el rayo de Röntgen y la primera película de los Lumière aparecieron en 1895, y el desarrollo de los primeros trabajos cinematográficos, como se evidencia por ejemplo en las fotografías stop-motion de Etienne-Jules Marey -un precursor del cine- están muy entrelazados con el conocimiento médico. En este sentido, en los albores del cine abundan las referencias a la medicina y a su práctica clínica. Uno de los primeros motivos del cine, la dislocación corporal, "aparentemente recordaban a marionetas o autómatas y de esa manera evocaban la vida mecánica, automática de las histéricas" (ibíd.: 112). Por ejemplo, en una película de Georges Méliès (1861-1938) titulada Turn of the Century Surgeon, de 1902, un cirujano quita y luego reemplaza cada uno de los miembros de su paciente. Este proceso está invertido en la película $\mathrm{La}$ muñeca (1919) de Ernst Lubitsch, en la que Hilarius, un fabricante de muñecas, funciona como una especie de fisiólogo que de manera milagrosa, miembro por miembro, ensambla muñecas de manera tan realista que parecen vivas, tornándose imposible distinguirlas de sus modelos, ya que uno y otro personajes son caracterizados por la misma actriz, Ossi Oswalda.

Samuel Beckett, a su vez, casi terminó haciendo una carrera en el cine -aunque más tarde, en 1956, sintió que "el cine fue asesinado en su cuna" (Beckett a Herbert White, 2 de julio de 1956, SBL II, 629). En marzo de 1936 el autor irlandés le escribió al famoso director ruso Sergei Eisenstein refiriéndose a sí mismo como "un cineasta digno". La carta de Beckett consistía principalmente de un curriculum vitae, y estaba escrita como una aplicación para estudiar en la Escuela Estatal de Cinematografía de Moscú. Beckett, nacido en 1906, había sido un entusiasta del cine desde su infancia en Dublín. Durante sus años de estudio en el Trinity College de Dublín y en su periodo como Lecteur en la École Normale Supérieure en París, de 1928 a 1930, miraba cine regularmente. Le gustaba particularmente el cine mudo de Buster Keaton y Charlie Chaplin, y fue en París donde habría visto varias películas vanguardistas, incluyendo Un perro andaluz (1928) y La edad de oro (1930) de Luis Buñuel y Salvador Dalí, cuya iconografía dejó huellas tanto en su teatro como en su prosa, según han argumentado críticos como Enoch Brater, entre otros. Aunque no tenemos certeza acerca de qué filmes expresionistas vio Beckett, es improbable que no haya visto El gabinete del Dr. Caligari, de Robert Wiene, finalizada en 1919 y estrenada en 1920. Lo mismo podría decirse con respecto a la película Nosferatu, de Murnau, de 1922, y de muchas producciones de Fritz Lang, como la serie de películas del Dr. Mabuse -Mabuse es un hipnotizador o médico de la mente que manipula a las personas para que actúen según su voluntad. Lo que tienen en común todas estas películas es que contienen, en primer lugar, muchas referencias médicas, como por ejemplo a la hipnosis y a otros desórdenes como el sonambulismo, la histeria, la discinesia, la amnesia y el ataque de nervios. En segundo lugar, todas esas películas comparten una forma de actuación muy estilizada que remite al cabaret y al music hall. Por último, estos filmes plasman una preocupación e inquietud temática dominante en cuanto a la pérdida de voluntad y autonomía: el sí mismo (cuerpo y mente) actuando según los dictámenes de fuerzas que están más allá de su propio control. 
Durante sus años en Dublín, Beckett frecuentaba los music hall Olympia y Gaiety, y en París solía ir al Bobino, un teatro de music hall cuyo estilo había nacido en el caféconcert. Usualmente iba solo a esos sitios, pero en una carta a Barney Rosset fechada en mayo de 1956 Beckett menciona su deseo de ver a su editor americano en París, y visitar juntos el "Bobino y cenar con baldes de Beaujolais y Sancerre"5 (de Beckett a Barney Rosset, 26 de mayo de 1956, SBL II, 620). Pero es también de las películas mudas que Beckett tomó el amor por las rutinas del circo y del music hall. Como he argumentado anteriormente, muchos comediantes de ese género continuaron su carrera de manera exitosa en el cine. Sabemos que Beckett vio al "Charlie Chaplin alemán", Karl Valentin, actuando en el Cabaret Benz en Munich en marzo de 1937, y que además unas semanas después lo conoció personalmente -el primero de abril- durante uno de sus viajes por Alemania. Beckett también conocía la iconografía de las películas expresionistas alemanas, y el 3 de noviembre de 1936, después de un paseo por la rivera del Elbe en Hamburgo, escribió en sus "Diarios alemanes":

Luego, caminando hacia Landungsbrücken y a través de Elbtunnel. Impresionante y espeluznante, especialmente el Fahrsschächten, pozos de acero con seis ascensores cada uno y escaleras caracol de las películas de los impresionistas alemanes. De alguna manera todo cinemático. Hordas de trabajadores portuarios volviendo a sus casas a lo lejos, llenando los ascensores y bajando las escaleras estrepitosamente. (“Diarios alemanes", 3/11/1936, citado en Viet 2009: 102)

En 1936 Beckett leyó el trabajo teórico de Rudolf Arnheim, Film als Kunst, que había sido publicado en Alemania en 1932 y que apareció en traducción inglesa en 1933 con el título Film. El libro de Arnheim podría ser considerado un compendio de la teoría modernista del cine que se desarrolló en varios países hacia finales de los años veinte y a comienzos de los treinta. Arnheim era un partidario del cine mudo, y para él la fuerza del cine como medio de expresión dependía precisamente de sus limitaciones. Arnheim escribió su libro en los comienzos del cine sonoro, pero defendía dos géneros distintivos de ese arte: un cine comercial que seguía los desarrollos tecnológicos, y el cine mudo de arte que tomaba su fuerza de las limitaciones del medio, postura que Beckett luego reflejaría en su correspondencia, en su estilo de escritura y en su película muda Film (1964), con Buster Keaton como protagonista. Beckett quería originalmente a Charlie Chaplin para el rol de "O" en la película, pero para decepción de Beckett, Chaplin rechazó la oferta.

Un ejemplo temprano del impacto del cine en la estética beckettiana fue "una parodia de El Cid, la tragedia en cuatro actos del siglo XVII de Pierre Corneille", en la que Beckett colaboró en 1931 con Georges Pelorson, cuando era profesor de francés en el Trinitiy College de Dublín. Por sugerencia de Beckett, la "pesadilla corneliana", tal como el programa la anunciaba, fue llamada Le Kid, como la famosa película de Chaplin The Kid de 1921, que era una de sus favoritas. El guion de la parodia no ha sobrevivido, pero Beckett describió la obra como "una combinación de Corneille y Bergson" (Knowlson 1996: 124). La crítica de la producción en el diario Irish Times caracterizó la obra como una mezcla de "clasicismo enfrentada al espejo distorsionante del expresionismo. Los héroes de Corneille de repente asumieron formas cómicas grotescas". 6 La influencia de las rutinas del music hall y las actuaciones estilizadas del cine mudo parecen haber desempeñado un papel importante en esta producción en la que el mismísimo Beckett actuó en el rol de don Diego, llevando un paraguas y un reloj alarma y moviéndose según los frenéticos dictámenes de un mecanismo acelerado, como anticipando la película de Chaplin Tiempos modernos, de 1936, en la que el protagonista está atrapado

\footnotetext{
5. La carta está reproducida en el libro de Carola Veit Kraft der Melone: Samuel Beckett im Kino (2009). 
en los engranajes frenéticos de una máquina. El estilo de actuación de Beckett podría quizás verse como una anticipación del automatismo, los errores y los tics de los personajes que el autor luego crearía.

Aquello que las enfermedades neurológicas tales como el mal de Parkinson, el síndrome de Tuourette y la epilepsia tenían en común, y lo que era visto como un recurso del humor negro en el cabaret y en las primeras películas, era la capacidad aparentemente mecánica del cuerpo para actuar fuera del reino del control de la propia conciencia. El trabajo de Henry Bergson La risa. Ensayo sobre el significado de la comicidad, publicado en 1899, le debía mucho a los descubrimientos neurológicos y su impacto en la cultura de la escena, por lo que autor argumentaba en su libro que "Las actitudes, gestos y movimientos del cuerpo humano son risibles en la medida exacta en que dicho cuerpo nos hace pensar en un simple mecanismo". (Bergson 2011: 24). ${ }^{7}$ El humor, repetía Bergson, surge de "un mecanismo funcionando detrás de lo vivo" (ibíd.: 27), ya que "un personaje cómico es generalmente cómico en la medida exacta en que se ignora a sí mismo" (ibíd.: 17) Esto hace que el sujeto aparezca como si estuviera privado de su libertad esencial:

Con su alada levedad esta alma comunica algo al cuerpo que anima: la inmaterialidad que pasa así a la materia es lo que llamamos la gracia. Pero la materia se resiste y se obstina. Atrae hacia sí misma, quisiera convertir a su propia inercia y rebajar al estado de automatismo la actividad siempre alerta de aquel principio superior. Quisiera fijar los movimientos del cuerpo, diversificados con inteligencia, en hábitos contraídos con estupidez, solidificar en muecas duraderas las cambiantes expresiones de la fisonomía, imprimir en toda la persona, en definitiva, una actitud tal que parezca estar hundida y absorbida en la materialidad de alguna ocupación mecánica en vez de renovarse sin cesar con el contacto de un ideal vivo. Ahí donde logra espesar exteriormente la vida del alma, fijar su movimiento, contradecir su gracia, la materia obtiene del cuerpo un efecto cómico. (lbíd.: 23)

Beckett leyó el texto de Bergson sobre la risa en 1930 o alrededor de esos años, y ciertos aspectos de su humor, así como de su actitud frente al lenguaje, la repetición y la compulsión, le deben mucho a dicha lectura. ${ }^{8}$ Aunque Beckett rechace la división entre espíritu y materia que Bergson toma de Descartes, se puede decir que su influencia es predominante, ya que no sólo los trabajos humorísticos de Beckett se inspiran en las ideas bergsonianas. ${ }^{9}$ El humor, después de todo, comienza a retroceder en la obra de Beckett después de Los días felices (1961), sin embargo el autor retiene su interés en las estructuras de comportamiento mecanizadas y osificadas, como se ve claramente en obras como Footfalls (1975), Rockaby (1980) y What Where (1983).

Los desórdenes neurológicos que daban forma al estilo performativo del music hall, del vaudeville, del cabaret y del cine, y en consecuencia el trabajo de Bergson, cuestionaban las ideas de soberanía e intencionalidad, y por lo tanto planteaban serias dudas sobre las nociones heredadas de subjetividad, sugiriendo que lo mecánico, lo automático y lo involuntario eran parte integral del ser. Después de todo, estas patologías

7. Utilizo la edición de Bergson, Henri (2011) La risa. Ensayo sobre el significado de la comicidad. Trad. Rafael Blanco. Buenos Aires: Ediciones Godot.

8. Aunque las notas sobre la lectura de Bergson no han sido recuperadas, podemos fecharlas alrededor de 1930, durante su breve período como Lecturer en francés en el Trinity College de Dublín. Beckett reveló su conocimiento sobre la obra de Bergson distinguendo en su curso de grado "entre el concepto de Bergson y el de Proust [,] y entre la actitud de Bergson ante el lenguaje y la de Gide" (Pilling 1997: 237, n. 25), como el ex estudiante de Beckett, Rachel Burrouws, lo atestigua en sus notas que se encuentran en los archivos del Trinity College de Dublín.

9. Para un análisis de Beckett como un escritor post-cartesiano, ver el texto de Maude, Beckett, Technology and the Body (2009). 
"sembraron serios cuestionamientos acerca de la viabilidad filosófica de la doctrina del libre albedrío" (Harris 1991: xvii). El mismo Beckett se había topado con el síndrome de Tourette hacia principios de los años treinta en su lectura del libro Degeneración, de Max Nordau, que había sido traducido al inglés en 1895 de la edición original de 1892, Entartung. Beckett tomó varias notas del libro de Nordau en su "Dream Notebook", que se encuentra en el archivo Beckett de la Universidad de Reading. En Degeneración Nordau describe a Emile Zola como un escritor con síndrome de Tourette, y le atribuye el estilo de su prosa a un desorden nervioso en el que abunda la coprolalia. Beckett leyó y tomó notas del siguiente pasaje del libro de Nordau:

Gilles de la Tourette ha acuñado el término "coprolalia" para designar las explosiones obsesivas de blasfemias y obscenidades que caracterizan una enfermedad descripta de manera exhaustiva por M. Catrou, y denominada "mal de tics convulsivos". M. Zola presenta un caso agudo de coprolalia. (Nordau 1986: 499).

El "Dream Notebook" de Beckett también contiene una referencia a la "ecolalia (repetición de palabras y sonidos)" (Beckett, en Pilling 1999: 91), que es otro famoso síntoma del síndrome de Tourette. Beckett leyó el siguiente pasaje en Degeneración:

una percepción suscita una apercepción que llama a la conciencia otras mil representaciones asociadas. El espíritu sano suprime las apercepciones o representaciones contradictorias o que no se concuerdan razonablemente con la primera apercepción; el imbécil no puede hacerlo. La simple consonancia determina el curso de su pensamiento; oye una palabra y experimenta la necesidad de repetirla una vez o varias veces: ecolalia; o bien palabra evoca en su conciencia palabras semejantes análogas a ella tan sólo por el sonido, no por el sentido, y en este caso piensa y habla en una serie de rimas absolutamente incoherentes; o bien las palabras tienen, además de la consonancia, alguna analogía muy remota de significación muy débil, y entonces se produce el juego de palabras o retruécano. $(\text { Nordau 1902: 103) })^{10}$

Los tics vocales y fónicos del síndrome de Tourette van de lo simple a lo complejo y pueden incluir aspiraciones, ladridos, malas palabras y ecolalia hasta llegar a frases cortas u oraciones. Lo mas llamativo de la descripción que hace Nordau de la ecolalia y de otras asociaciones de palabras "imbéciles", según dice, es su proximidad al lenguaje poético: a la polisemia, la rima, el ritmo y el juego de palabras.

Los personajes de Beckett, sea los de sus trabajos en prosa o los de su teatro, son casi únicos dentro de la literatura modernista por su propensión hacia los movimientos convulsivos o "clawnescos" que Charcot asociaba con la histeria y la epilepsia, y que también se presentaban en otros desórdenes neurológicos investigados en el hospital de la Salpêtrière, como por ejemplo el mal de Parkinson y el síndrome de Tourette. En las páginas y los escenarios beckettianos la renguera, los tics, las convulsiones y los temblores hacen su famosa aparición cuando Pozzo en Esperando a Godot (1953) y Clov en Fin de partida (1957) sufren distintos grados de acatisia, la imposibilidad de sentarse. Beckett también tomó nota de esta enfermedad en su "Psychology Notebook" de los años treinta, que se encuentra en el Trinity College de Dublín. Además, aquello que es distintivo de la obra de Beckett es que estos llamados automatismos, que al principio del siglo XIX la medicina atribuía a hábitos patológicos y a la memoria motriz, también se hallan presentes en el lenguaje de su obra, en el que abundan no sólo la coprolalia y

10. Utilizo la edición de Nordau, Max (1902) Degeneración. Trad. Nicolás Salmerón y García. Madrid: Librería de Fernando Fé, Saenz de Jubera Hermanos. 
ecolalia -después de todo Beckett quizás sea el escritor modernista más coprológicosino también otras formas de enunciación que no parecen pertenecer simplemente al campo del discurso intencional. En cuanto a esto, su escritura nos lleva a la observación de la mente que Bergson realiza en Matter and Memory, de 1896 (traducida en 1911) acerca de cómo el automatismo de la memoria motriz se extiende mucho más lejos de lo que uno ve a primera vista. Bergson escribe:

Hemos visto a dementes producir respuestas inteligentes a una serie de preguntas que no comprendían: el lenguaje funcionaba en ellos a la manera de un reflejo. A afásicos incapaces de pronunciar espontáneamente una palabra acordarse sin error las letras de una melodía cuando la cantan. O también recitarán corrientemente una plegaria, la serie de los números, la de los días de la semana y los meses del año. (Bergson 2006: 104) ${ }^{11}$

En otras palabras, también el lenguaje puede funcionar como un mero automatismo, algo que nos lleva a una de las preocupaciones centrales de la obra de Beckett, a saber, que el lenguaje más que dar cuenta de una subjetividad, usualmente parece hablar por sí mismo. Este punto de vista de Tourette sobre el lenguaje está sorprendentemente presente, por ejemplo, en el famoso monólogo de Lucky en Esperando a Godot (que sobre el escenario presenta los temblores y tics de los desórdenes neurológicos), o en la compulsiva y convulsiva prosa de El innombrable, obra en la que el lenguaje "sale de mí, me llena, clama contra mis paredes, no es la mía, no puedo detenerla, no puedo evitar que me desgarre, me sacuda, me asedie. No es la mía, no tengo, no tengo voz y debo hablar" (Beckett, 1983: 56). ${ }^{12}$ El lenguaje, en otras palabras, se vuelve un acto patológico, que está más allá del control intencional del hablante. La Boca en Not I, de 1972, de repente siente " ¡sus labios moviéndose!... como si hasta entonces no hubiera... y no sólo los labios... las mejillas... la mandíbula... toda la cara... todos esos... ¿qué?... ¿la lengua?... sí... la lengua dentro de la boca... todas esas contorsiones sin las cuales... no es posible hablar..." (2006: 421). ${ }^{13}$ Beckett le escribió a Alan Schneider, el más destacado director estadounidense de sus obras, el 16 de octubre de 1972, diciendo que el discurso de la Boca era "un fenómeno puramente oral, sin control mental o comprensión, sólo escuchado a medias. El órgano y su función desaparecen”. De la voz de la Boca, Beckett declaró: "La escucho sin aliento, urgente, febril, rítmica, jadeando, sin preocupación por su inteligibilidad. Ligada menos a la comprensión que a los nervios de la audiencia, que de alguna manera comparte su desconcierto" (Harmon 1998: 283). Este es Beckett poniendo en escena el lenguaje como una perturbación parasitaria: como un hábito patológico.

Una obra que se focaliza centralmente en las diversas manifestaciones del automatismo lingüístico es Los días felices, que Beckett escribió en inglés entre 1960 y 1961. El texto presenta dos protagonistas, Winni, una mujer "de unos cincuenta años", y Willie, un hombre "en sus sesenta años", clavados en un paisaje desértico bajo una luz cegadora. (Beckett 2010a: 5). En el primer acto de la obra, Winnie está enterrada hasta la cintura en un montículo; en el segundo acto ella aparece enterrada hasta el cuello, virtualmente devorada por la tierra. Su marido, Willie, aunque apenas se mueve todavía puede gatear. Como no podía ser de otra manera, el título de la obra fue tomado de una canción de music hall compuesta por Milton Ager y Jack Yellen: "Los días felices están aquí otra vez".

11. Utilizo la edición de Bergson, Henri (2010) Materia y memoria. Trad. Pablo Ires. Buenos Aires: Cactus.

12. Utilizo la edición de Beckett, Samuel (1983). El innombrable. Trad. R. Santos Torroella. Buenos Aires: Hyspamérica.

13. Utilizo la edición de Beckett, Samuel (2006) No yo, en Teatro reunido. Trad. José Sanchis Sinisterra, Ana María Mouix y Jenaro Talens. Buenos Aires Tusquets. 
Como en gran parte de la obra de Beckett, la obra es notable por el hecho de que en ella es muy poco lo que sucede. En palabras de Daniel Albright, "Los días felices es en algún punto posteatral de la manera en que lo es Fin de partida, en el hecho de que el concepto del evento parece pertenecer mucho más al pasado que al presente" (Albright 2003: 75). Una campana suena seis veces tanto para despertar a Winnie como para evitar que se quede dormida (además se pueden reconocer varias referencias a las observaciones de Pavlov, como lo he mencionado en otro de mis trabajos). ${ }^{14}$ Winnie se cepilla los dientes y el cabello y recurre a once objetos banales que lleva en su bolsa de mano: su lápiz labial, un cepillo para el pelo, un cepillo de dientes, unos anteojos, una lima de uñas, un espejo, un sombrero con una pluma, una lupa, un revólver, una cajita de música y una botella de alixir. Además Winnie de manera repetida se olvida y distorsiona las citas de sus clásicos: Shakespeare, Thomas Gray, Omar Khayyam, Robert Browning, Milton, Keats, Charles Wolfe, Yeats y Herrick.

Entre las primeras líneas de Winnie aparece una plegaria inaudible de la de se escuchan sólo las últimas palabras "Por Cristo, Nuestro Señor, amén" (Beckett 1999: 131). ${ }^{15}$ Como dice Stan Gontarski, "Winnie nunca escucha su plegaria. El rezo es simplemente parte de su ritual matutino, de su hábito, que Beckett nos ha recordado que es un gran lastre" (Gontarski 1985: 74). Ruby Cohn describe la plegaria de Winnie como "automática", y agrega que "ella invoca a la deidad meramente como una exclamación" (Cohn 1973: 179). La plegaria y las exclamaciones de Winnie tales como "Dios" ante la vista de una hormiga llevando un huevo funcionan como un acto reflejo, como un hábito desprovisto de contenido semántico (ibíd.: 18). Uno de los problemas mas grandes que tuvo Beckett al escribir la obra, como él mismo le dice a Alan Schneider en una carta de diciembre de 1960, fue la cuestión de "cómo hacer [que Winnie] hablara sola todo el tiempo en el escenario sin que se dirija a ella misma ni a la audiencia" (Harmon 1998: 77). El efecto notable de la obra viene precisamente de la disparidad entre el espectáculo del escenario y las palabras que se comunican: "Esta irrelevancia calculada de lenguaje hasta las imágenes visuales está mutuamente desacreditada, este tintineo, esta inconsistencia no pueden ser paliadas a través de ninguna estrategia de interpretación" (Albright 2003: 66).

Algo bastante similar se puede aplicar a las numerosas citas mal dichas. Cuando en 1971 Beckett estaba dirigiendo a Eva Katharina Schultz como Winnie en Glückliche Tage, la traducción alemana de la obra, en el teatro Schiller de Berlín, "él quería algo cercano a un cántico para las citas" (Cohn 1973: 189). Las citas distorsionadas de Winnie, como por ejemplo la línea que repite de Cimbelino, "No temas ya el calor del sol", o la referencia a la "Oda a una perspectiva lejana de la universidad de Eton", "muriéndose de risa... algo muriéndose de risa en el más cruel infortunio", funcionan aquí a la manera de cánticos culturales o mantras: son las líneas clave de la literatura de Occidente, pero para Winnie su significado hace ya mucho tiempo que está destruido (Beckett 1999: 177). Las distorsiones de las citas funcionan como un ejemplo del cambio generado por el hábito a través de la repetición, lo cual erosiona de manera gradual las líneas del verso y la prosa, alterando su forma. Pero también son un ejemplo de la naturaleza mecánica del mismo lenguaje, osificado como un cántico y como un mera estructura sonora. En su ensayo final dedicado a la obra de Beckett, Deleuze escribe que las palabras "están tan cargadas de cálculos y significaciones, y también de intenciones y recuerdos personales, de viejos hábitos que los cimientan, que su superficie se vuelve a cerrar apenas abierta. Se pega. Nos aprisiona y nos asfixia" (Deleuze 1995: 22). Era importante para Beckett que esas características del lenguaje -lo habitual, calcificado- fueran expresadas sobre el escenario. En agosto de 1961 Beckett le pidió a Alan Schneider, quien

\footnotetext{
14. Ver el texto de Maude "Pavlov and other animals in Samuel Becket" (2013).

15. Utilizo la edición de Beckett, Samuel (1999) Los días felices. Trad. Antonia Rodríguez Gago. Madrid: Cátedra.
} 
estaba dirigiendo la premier americana de la obra, que mantenga "el mismo tono a través de toda la pieza, perfeccionando la cuestión mecánica, sin emoción... En una palabra aquí pido que se mantenga la monotonía vocal y que se confíe en los ritmos del discurso y en las complejidades gestuales del mismo" (Harmon 1998: 95). Luego, en la misma carta, Beckett se refería a lo "anormal del discurso" de Winnie, y diez años después, mientras dirigía a Eva Katharina Schultz le aconsejó a la actriz que "hablara con normalidad, pero que se mantuviera curiosamente distante, impersonal" (Ben-Zvi 1990: 22). Las citas de Winnie constituyen en simples escombros verbales, y en este sentido apenas se diferencias de los objetos en su bolsa, que tienen la función de actuar como reliquias "del viejo estilo". Con el fin de pasar el tiempo, Winnie contempla la posibilidad de hacer un listado de sus pertenencias: "Queda la bolsa, por supuesto... ¿Podría enumerar su contenido?”, esto nos lleva al inventario de Malone de la trilogía (Beckett 1999: 215). Aquí, como un muchas de las citas distorsionadas de Winnie, la cuestión de hacer listados e inventarios -que es la forma más primitivas de alfabetismo- constituye la estética beckettiana de la degradación. Stan Gontarski argumenta que las palabras finalmente "tienen muy poco significado para [Winnie], aparte de mantener el sonido" (Gontarski 1985: 74). Las palabras funcionan como lo hacen los gritos afásicos de Bergson: como una repetición de una estructura de ritmo y sonido. Beckett quería que sobre el escenario se reflejara una "monotonía simétrica" similar: "la clase de chabacanería que aparece en un musical o una pantomima de tercera, esa característica del pompier, una risible mala imitación de algo serio" (Harmon 1998: 94).

Los desórdenes neuronales que presentan un automatismo motriz o lingüístico confunde y hace colapsar las distinciones entre la acción voluntaria y la involuntaria, entre la capacidad y la discapacidad, entre las formaciones biológicas y las culturales, entre la habilidad motriz y la actividad lingüística, entre la neurología y la psicología, entre el lenguaje poético y las patologías del lenguaje, y finalmente entre el cuerpo y la mente. El estatus de los desórdenes tales como el de Tourette, así también como en casos límite entre varias formas de clasificar y pensar acerca de la subjetividad y el comportamiento pueden en parte ayudar a explicar la importancia con la que los "síntomas" de esos desórdenes aparecen en la obra de Beckett. Sin embargo, los textos que he estado discutiendo además ofrecen un ejemplo de los modos en que la escritura modernista, tanto en lo que concierne a la forma como al contenido, responde a los nuevos descubrimientos médicos, en este caso a partir de reevaluar nuestra relación con el lenguaje mismo. Como dijo una vez Steven Connor, "no podemos decir de dónde viene el lenguaje, o dónde este se relaciona con las furiosas contorsiones que vemos delante nuestro" (Connor 1988: 163). Debido a que se concibe el lenguaje como la expresión de un sujeto y de una intencionalidad, cuando buscamos sus orígenes a menudo nos encontramos con una performance sin sujeto y sin intención, una asombrosa ausencia y vacío del sí mismo. Esto es paradigmático en la figura de la Boca en Not I, que es al mismo tiempo presencia y vacío, otro "agujero olvidado" (Beckett 2009: 85) y, al mismo tiempo el asunto mismo, la materialización del lenguaje. 


\section{Q Bibliografía}

»Albright, Daniel (2003) Beckett and Aesthetics. Cambridge: Cambridge University Press.

»Arnheim, Rudolf(1933) Film. Trans. L. M. Sieveking and lan D. F. Morrow. London: Faber \& Faber.

» Beckett, Samuel (1979) The Beckett Trilogy: Molly, Malone Dies and The Unnamable. London: Picador.

» Beckett, Samuel (1999) Los días felices. Trad. Antonia Rodríguez Gago. Madrid: Cátedra.

»Beckett, Samuel (2006) No yo, en Teatro reunido. Trad. José Sanchis Sinisterra, Ana María Mouix y Jenaro Talens. Buenos Aires Tusquets.

» Beckett, Samuel (1983). El innombrable. Trad. R. Santos Torroella. Buenos Aires: Hyspamérica.

"Beckett, Samuel (2011) The Letters of Samuel Beckett 1941-1956, ed. George Graig, Martha Dow Fehsenfeld, Dan Gunn and Lois More Overbeck. Cambridge: Cambridge University Press.

»Ben-Zvi, Linda (ed.) (1990) Women in Beckett: Performance and Critical Perspectives. Urbana and Chicago: University of Illinois Press.

» Bergson, Henri (2010) Materia y memoria. Trad. Pablo Ires. Buenos Aires: Cactus.

»Bergson, Henri (2011) La risa. Ensayo sobre el significado de la comicidad. Trad. Rafael Blanco. Buenos Aires: Ediciones Godot.

»Cohn, Ruby (1973) Back to Beckett. Princeton: Princeton University Press.

»Connor, Steven (1988) Samuel Beckett: Repetition, Theory and Text. Oxford: Balckwell.

"Deleuze, Gilles (1995) “The Exhausted”, trans. Anthony Uhlmann, SubStance, 78.

»Didi-Huberman, Georges (2007) La invención de la histeria. Charcot y la iconografía de la Salpêtrière. Trad. Tania Arias y Rafael Jackson. Madrid: Cátedra.

»Epstein, Jean "Magnification", French Film Theory and Criticism: A History/ Anthology 1907-1939. Volume I, ed. Richard Abel. Princeton: Princeton University Press, pp. 235-41.

» Freud, Sigmund and Breuer, Joseph (1980) Studies on Hysteria, trans. James and Alix Strachey. Harmondsworth: Penguin.

» Gontarski, S. E. (1985) The Intent of Undoing in Samuel Beckett's Dramatic Texts. Bloomington: Indiana University Press.

» Gontarski, S. E. (2001) Why The French Love Jerry Lewis: From Cabaret to Early Cinema. Stanford: Stanford University Press.

" Gontarski, S. E. (2004) "From Charcot to Charlot: unconscious imitation and spectatorship in French cabaret and early cinema", in Mark S. Micale (ed.), The Mind of Modernism. Stanford: Stanford University Press, pp. 93-124.

" Hacking, Ian (1998) Mad Travelers: Reflections on the Reality of Transient Mental 
Illness. Cambridge, MA: Harvard University Press.

» Harmon, Maurice (ed.) (1998) No Author Better Served: The Correspondence of Samuel Beckett \& Alan Schneider. Cambridge, MA: Harvard University Press.

" Harris, Ruth (1991) "Introduction", in Jean-Martin Charcot, Clinical Lectures on Diseases of the Nervous System, ed and intro. Ruth Harris. London: Tavistock/ Routledge, pp. ix-Ixviii.

"Knowlson, James (1996) Damned to Fame: The Life of Samuel Beckett. London: Bloomsbury.

» Maude, Ulrika (2009) Beckett, Technology and the Body. Cambridge: Cambridge University Press.

" Maude, Ulrika (2013) "Pavlov's dogs and other animals in Samuel Beckett", in Mary Bryden (ed.), Beckett and Animals. Cambridge: Cambridge University Press.

» Micale, Mark S. (1995) Approaching Hysteria: Disease and Its Interpretations. Princeton: Princeton University Press.

» Nordau, Max (1902) Degeneración. Trad. Nicolás Salmerón y García. Madrid: Librería de Fernando Fé, Saenz de Jubera Hermanos.

» Pilling, John (1997) Beckett before Godot. Cambridge: Cambridge University Press.

» Pilling, John (1999) Beckett's Dream Notebook, ed., annot. And intro. Essay John Pilling. Reading: Beckett International Foundation.

» Porter, Roy (1999) The Greatest Benefit to Mankind: A Madiacl History of Humanity from Antiquity to the Present. London: Fontana.

»Veit, Carola (2009) Kraft der Melone: Samuel Beckett im Kino. Berlin: Deutsche Kinematek Museum für Film und Fehrnsehen. 
